\title{
Etat actuel de la pollution du lait et des produits laitiers par les résidus de composés organo-chlorés
}

\author{
par \\ L. RICHOU-BAC \\ Laboratoire Central de Recherches Vétérinaires d'Alfort \\ 22, rue Pierre-Curie (94) Maisons-Alfort \\ (Service d'Hygiène Alimentaire)
}

La pollution du lait et de ses dérivés par les résidus de pesticides préoccupe les milieux scientifiques et économiques internationaux depuis déjà une dizaine d'années.

Plus récemment, ont été mis en évidence des résidus de polluants d'origine industrielle : les diphényles polychlorés. Enfin, les résidus de certains anthelminthiques ont été décelés dans le lait d'animaux soumis à des traitements prophylactiques intensifs.

Parmi tous ces composés chimiques de synthèse, les pesticides organo-chlorés apparaissent encore, sans aucun doute, comme les plus inquiétants.

Rappelons qu'en France, les premiers sondages ont été réalisés à partir de 1965-66 (Mestres R., 1966 [1], Thieulin G. et al., 1966 [2], Viel et al., 1967 [3]). Ces premières recherches mirent alors en évidence le rôle prédominant de l'HCH en tant que résidu.

A l'étranger, le problème semble assez différent. Les premiers travaux de prospection réalisés dès 1961 au Danemark et en 1962 aux Etats-Unis, furent suivis par un grand nombre de contrôles systématiques qui mirent en évidence l'importance des résidus de DDT et de ses dérivés.

Les résidus de diphényles polychlorés (PCB) composés utilisés dans l'industrie électrique, mécanique, chimique, depuis de très nombreuses années, ont été mis en évidence pour la première fois dans le gibier et certains poissons par Jensen, à Stockholm, en 1966 [4]. Depuis cette date ils ont été décelés à plusieurs reprises dans certains laits (en Allemagne et aux Etats-Unis).

Enfin, des résidus de fasciolicides organo-chlorés (oxyclozanide, ménichlopholane) ont été dosés récemment dans le lait de bovins 
soumis à des traitements antiparasitaires continus (Heeschen et al., 1971 [5]).

Nous examinerons, en premier lieu, le niveau de contamination actuel des principaux composés en cause. Ces composés en effet sont pour la plupart doués d'une très grande stabilité et leur pouvoir lipophile élevé les fait s'accumuler et persister dans un grand nombre de denrées riches en lipides dont le lait.

Nous étudierons ensuite quels enseignements il est possible de tirer des résultats observés, en particulier l'évaluation des risques auxquels s'exposent l'homme ou l'animal, considérés comme consommateurs des denrées contaminées.

\section{I. - NIVEAU DE CONTAMINATION}

\section{1) Méthode analytique}

Les analyses s'effectuent par chromatographie en phase gazeuse après séparation des matières grasses et purifications préalables (double partage).

Appareillage

1) Détecteur à capture d'électron : source Ni 63.

2) Détecteur à flamme modifiée - pour la vérification éventuelle de la présence de résidus de composés organo-phosphorés ou organosoufrés.

3) Colonnes : deux colonnes obligatoires pour le contrôle : phases stationnaires DOW II et QF 1.

Trois colonnes pour les cas difficiles exigeant des recherches supplémentaires: DOW II, QF 1, OV 225,

ou DOW II, OV 17, OV 225.

Le couplage chromatographie en phase gazeuse/spectrométrie de masse doit permettre, dans un proche avenir, l'identification rapide des composés mal définis qui sont parfois rencontrés dans les analyses de produits laitiers.

\section{2) Modes de contamination}

Les contaminations s'effectuent en général par la voie digestive.

De nombreuses expérimentations ont été réalisées, tant en France qu'à l'étranger, dans le but de déterminer aussi précisément que possible les conditions exactes de la pollution. 
En France, en ce qui concerne les résidus de pesticides organochlorés, trois éléments déterminants sont à considérer :

A) Les traitements des Étables et des locaux de Stockage deS ALIMENTS

Les composés en cause sont l'HCH $\alpha, \mathrm{l}^{\prime} \mathrm{HCH} \beta, \mathrm{l}^{\prime} \mathrm{HCH} \gamma$ (lindane).

Les blanchissements des locaux de stabulation ont été la source principale de la contamination par l'HCH. L'écoulement de certains stocks après l'interdiction de 1969, a malheureusement prolongé la présence de ce composé, dont un isomère $(\beta)$ possède une rémanence et une résistance des plus marquées.

Le lindane (isomère $\gamma$ ) employé un certain temps après 1969 ne semble plus poser de problème actuellement sous cette forme de contamination.

\section{B) L'ALIMENTATION}

Les composés en cause sont l' $\mathrm{HCH} \alpha, l^{\prime} \mathrm{HCH} \gamma$ (lindane), l'heptachlore, l'aldrine, la dieldrine, l'HCB.

La contamination des végétaux entrant dans la composition de l'aliment peut intervenir directement sur les taux résiduels observés. Ainsi peuvent être mis en cause : les céréales, les tourteaux, les aliments concentrés, certains fourrages ou aliments particuliers (racines d'endives). Ce sont, en général, les tourteaux et les concentrés qui apportent des contaminants de type $\mathrm{HCH}$, par un processus extérieur à l'exploitation, disparate, fonction du hasard des achats.

L'époxyde de l'heptachlore provient lui directement de l'utilisation en alimentation animale des pulpes de betteraves (Nord, Nord-est) et de maïs fourrage (Ouest, Sud-ouest). Il ne faut pas oublier que les traitements moyens phyto-sanitaires qui s'effectuent avec 4 à $6 \mathrm{~kg}$ de matière active à l'ha polluent le sol durant plusieurs années.

- L'heptachlore, l'aldrine, la dieldrine, conservent leur activité presque intégrale 7 ans après l'application (95 p. 100). Ils présentent encore une action relativement forte $(25$ p. 100) 9 à 13 ans après les premiers traitements.

- La dieldrine peut provenir des traitements de prairie à l'engrais aldriné ( 4 à $5 \mathrm{~kg}$ de M.A. à l'ha). En fait, ce composé se retrouve dans certains tourteaux ou concentrés contaminés la plupart du temps dans des locaux de stockage défectueux.

L'hexachlorobenzène (HCB) enfin, fongicide de synthèse, est très peu employé en France depuis quelques années. C'est plutôt comme sous-produit de fabrication du PCNB (pentachloronitrobenzène ou quintozène) qu'il peut être considéré comme polluant du lait dans certaines régions du Nord de la France. Cette pollution se ferait par les racines d'endives traitées qui sont données aux vaches laitières régulièrement dans de nombreuses exploitations. 
En fait, des informations récentes permettent d'affirmer que l'HCB peut être considéré, à l'image des $\mathrm{PCB}$, comme un polluant industriel. Il semble en effet couramment utilisé dans l'industrie chimique (élastomères), ceci expliquerait sa présence fréquente dans la chaîne alimentaire et l'environnement.

C) LES INTERVENTIONS THÉRAPEUTIQUES VÉTÉRINAIRES semblent enfin être la cause directe de contaminations dues au lindane ( $\mathrm{HCH} \gamma)$. Les traitements des ecto-parasites (particuliers chez les ovins et caprins) déterminent une augmentation rapide des taux résiduels (20 à 25 fois plus que la contamination de départ) qui s'annule d'ailleurs assez rapidement 3 à 4 semaines après l'application.

Ces actions thérapeutiques présentent donc un inconvénient certain, beaucoup plus important que les traitements phyto-sanitaires correspondants avec ce même produit.

Ajoutons qu'en ce qui concerne les diphényles polychlorés, les formes de la contamination peuvent être très variées. Ces composés sont présents en effet dans un très grand nombre de produits industriels (notamment peintures, laques, vernis). Les divers cas de contamination du lait signalés sont dus à des pollutions accidentelles d'eau de boisson (abreuvoirs ou rivières) ou d'aliments (grains ensilés).

\section{3) Niveaux de contamination actuels}

A) RÉSIDUS DE PESTICIDES ORGANO-CHLORÉS

En France, depuis 5 ans, les variations des taux résiduels des pesticides organo-chlorés, dans les produits laitiers que nous avons plus particulièrement analysés, amènent les conclusions suivantes :

1) La pollution générale en $\mathrm{HCH}$ total est en diminution régulière, quoique légèrement stabilisée depuis quelques mois. Les taux résiduels de l'isomèreß varient peu, ceux de l'isomère $\gamma$ (lindane) demeurent, sauf cas exceptionnels, toujours extrêmement faibles (voir tab. 1 et fig. 1).

2) L'époxyde de l'heptachlore persiste et augmente régulièrement; sa présence est particulièrement importante dans les régions du Nord et du Nord-est.

3) La contamination par la dieldrine a sensiblement la même importance et la même répartition que celle de l'époxyde de l'heptachlore.

4) Les résidus d'hexachlorobenzène (HCB) sont en général assez faibles et inconstants.

Les résultats des analyses des produits laitiers examinés au Laboratoire Central de Recherches Vétérinaires d'Alfort pour les années 1970-71 (voir tab. 2) sont le reflet des autres enquêtes effectuées en France, en particulier celles de la profession laitière et de l'Institut Technique de l'Elevage bovin. 


\section{$T A B L E A U 1$}

Résidus de pesticides organo-chlorés - Moyenne arithmétique (en ppm (mg/kg) M.G.) - Produits laitiers Laboratoire Central de Recherches Vétérinaires d'Alfort - Service Hygiène Alimentaire

\begin{tabular}{|c|c|c|c|c|c|}
\hline & 1967 & 1968 & 1969 & 1970 & 1971 \\
\hline $\mathrm{HCH}(\alpha)$ & 0,73 & 0,64 & 0,46 & 0,21 & 0,16 \\
\hline $\mathrm{HCH}(\beta)$ & 0,16 & 0,11 & 0,10 & 0,08 & 0,09 \\
\hline $\mathrm{HCH}(\gamma)$ & 0,03 & 0,03 & 0,03 & 0,03 & 0,04 \\
\hline $\mathrm{HCH}$ total & 0,92 & $\begin{array}{c}0,78 \\
(0,72)\end{array}$ & $\begin{array}{c}0,59 \\
(0,44)\end{array}$ & $\begin{array}{c}0,32 \\
(0,50)\end{array}$ & $\begin{array}{c}0,29 \\
(0,28 \text { à } 0,37)\end{array}$ \\
\hline HE.H & 0,02 & 0,025 & 0,03 & $\begin{array}{c}0,06 \\
(0,09)\end{array}$ & $\begin{array}{c}0,10 \\
(0,12)\end{array}$ \\
\hline D.A. & 0,02 & 0,03 & 0,04 & $\begin{array}{c}0,05 \\
(0,04)\end{array}$ & $\begin{array}{c}0,09 \\
(0,07)\end{array}$ \\
\hline $\mathrm{DDT}+\mathrm{M}$ & $\mathrm{T}$ & 0,02 & $\mathrm{~T}$ & $\mathrm{~T}$ & $\mathrm{~T}$ \\
\hline
\end{tabular}

$\mathrm{HCH}=$ Hexachlorocyclohexane.

$\mathrm{HE}=$ Epoxyde de l'Heptachlore

$\mathrm{H}=$ Heptachlore.

$\mathrm{D}=$ Dieldrine.

A $\quad=$ Aldrine pp' DDT = isomère pp' du 1,1,1 trichloro 2, 2 bis chlorophényléthane métabolites du pp' DDT = DDE - DDD

$\mathrm{T}=$ Traces $(\leqslant 0,001 \mathrm{ppm})$.

Entre parenthèses : résultats (moyennes) d'autres laboratoires d'analyse français : Industrie Laitière de Douai - I.N.R.A. Versailles. 
TABLEAU 2

Résidus de pesticides organo-chlorés - Résultats en $\mathrm{ppm}(\mathrm{mg} / \mathrm{kg})$ par rapport à la matière grasse Laboratoire Central de Recherches Vétérinaires d'Alfort - Service Hygiène Alimentaire

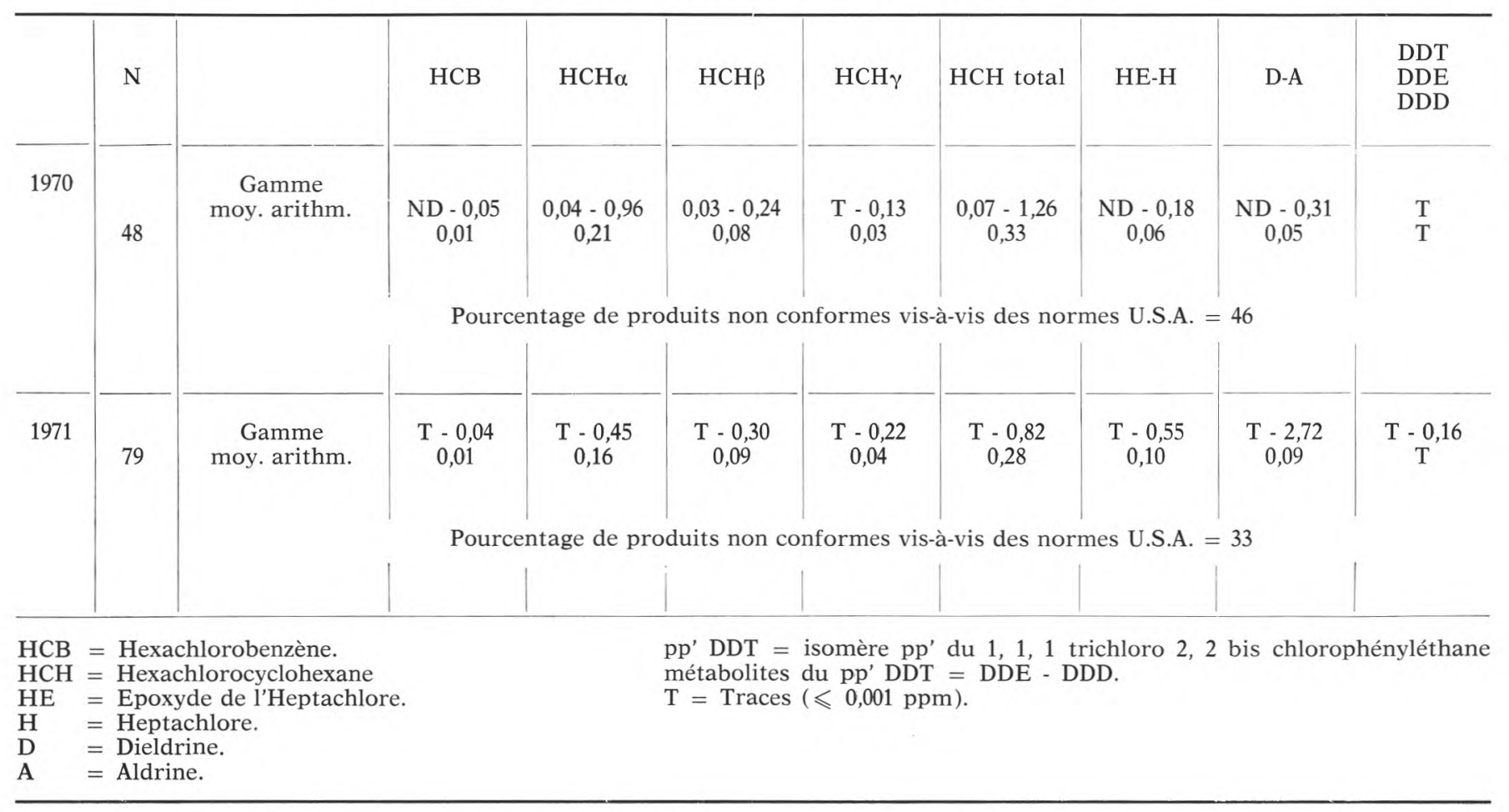



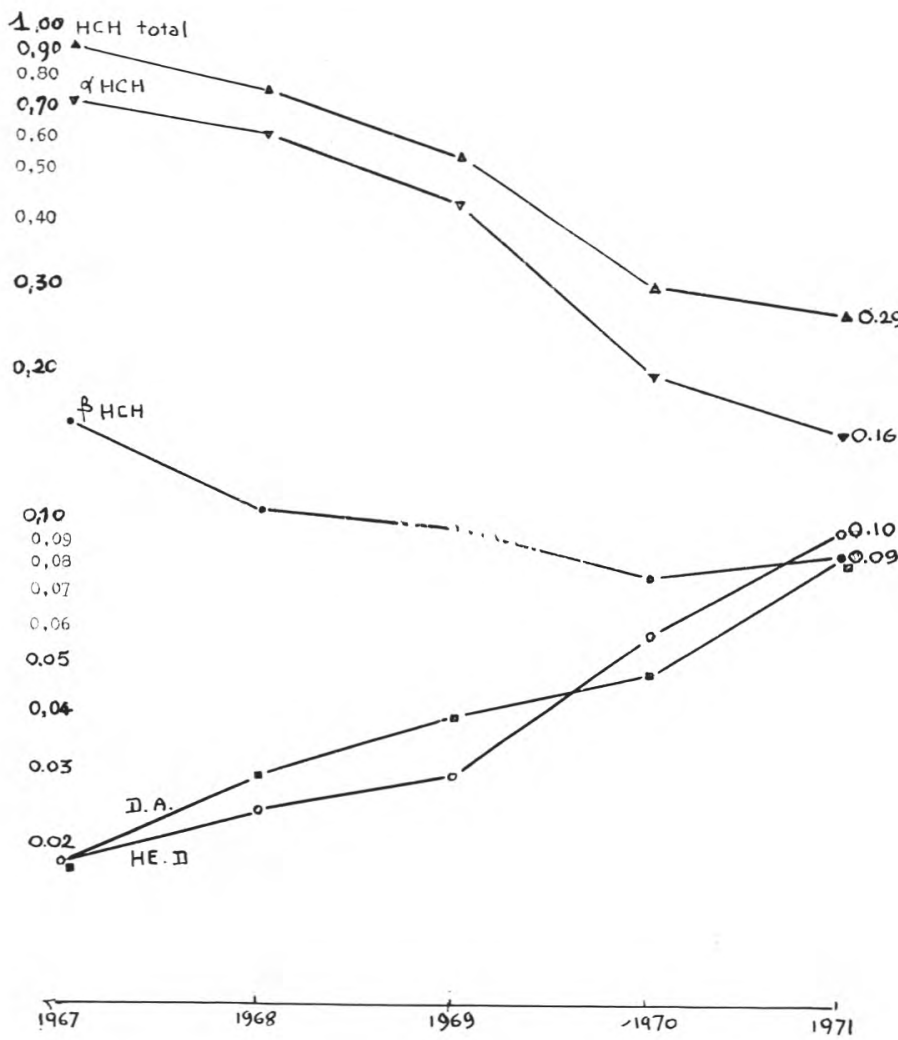

fig. 1

Résidus de pesticides organo-chlorés - Produits laitiers (ppm mg/kg M.G.)
$\Delta \mathrm{HCH}$ total
○ Heptachlore + Epoxyde
$\nabla \mathrm{HCH} \alpha$
- Dieldrine + Aldrine

Nos analyses ont porté presque exclusivement sur des produits laitiers (fromages en grande majorité).

Sur les 127 échantillons étudiés (1970-71), 45 sont des fromages fermentés à pâte molle (Brie, Camembert, Pont l'Evêque), 41 des fromages fermentés à pâte pressée (Saint-Paulin, Reblochon, Cantal, Emmental, Comté), 15 des fromages fondus. Egalement 26 crèmes et beurres, de provenances diverses, ont été analysés.

Les origines de ces échantillons sont assez variées, surtout les départements de l'Eure (27), du Puy-de-Dôme (63), de la Haute-Savoie (74), de la Seine-et-Marne (77) et des Vosges (88). 


\section{TABLEAU 3}

Résidus de pesticides organo-chlorés - Beurres - Moyennes en ppm (mg/kg)

\begin{tabular}{|c|c|c|c|c|c|c|}
\hline Pays & Année & $\begin{array}{c}\text { Nombre } \\
\text { d'échantillons }\end{array}$ & $\begin{array}{c}\text { DDT } \\
+ \text { métabolites }\end{array}$ & $\begin{array}{l}\mathrm{HCH} \\
\text { total }\end{array}$ & $\begin{array}{l}\text { Dieldrine } \\
+ \text { Aldrine }\end{array}$ & $\begin{array}{l}\text { Heptachlore } \\
\text { Epox. + Hept. }\end{array}$ \\
\hline Australie & $\begin{array}{l}1962-3 \\
1964 \\
1965 \\
1966 \\
1967\end{array}$ & $\begin{array}{l}12 \\
45 \\
45 \\
53 \\
35\end{array}$ & $\begin{array}{l}0,28 \\
0,40 \\
0,25 \\
0,54 \\
0,27\end{array}$ & $\begin{array}{l}0,007 \\
0,02 \\
0,02 \\
0,01 \\
0,01\end{array}$ & $\begin{array}{l}0,01 \\
0,01 \\
0,01 \\
0,01 \\
0,01\end{array}$ & $\begin{array}{l}- \\
- \\
-\end{array}$ \\
\hline Danemark & $\begin{array}{l}1962-3 \\
1964 \\
1965 \\
1966 \\
1967\end{array}$ & $\begin{array}{l}20 \\
30 \\
24 \\
24 \\
12\end{array}$ & $\begin{array}{l}- \\
0,05 \\
0,05 \\
0,04 \\
0,04\end{array}$ & $\begin{array}{l}0,02 \\
0,05 \\
0,04 \\
0,05 \\
0,05\end{array}$ & $\begin{array}{l}0,035 \\
0,04 \\
0,03 \\
0,03 \\
0,03\end{array}$ & $\begin{array}{l}- \\
- \\
-\end{array}$ \\
\hline Grande-Bretagne & $\begin{array}{l}1962-3 \\
1964 \\
1965 \\
1966 \\
1967\end{array}$ & $\begin{array}{l}15 \\
18 \\
18 \\
13 \\
22\end{array}$ & $\begin{array}{l}0,03 \\
0,05 \\
0,04 \\
0,05 \\
0,05\end{array}$ & $\begin{array}{l}0,09 \\
0,05 \\
0,04 \\
0,07 \\
0,07\end{array}$ & $\begin{array}{l}0,07 \\
0,03 \\
0,03 \\
0,02 \\
0,03\end{array}$ & $\begin{array}{l}- \\
- \\
-\end{array}$ \\
\hline Hollande & $1967-8$ & 359 & 0,12 & 0,06 & 0,05 & 0,02 \\
\hline Irlande & $\begin{array}{l}1966 \\
1967\end{array}$ & $\begin{array}{l}5 \\
6\end{array}$ & $\begin{array}{l}0,10 \\
0,05\end{array}$ & $\begin{array}{l}0,07 \\
0,05\end{array}$ & $\begin{array}{l}0,03 \\
0,02\end{array}$ & - \\
\hline Nouvelle-Zélande & $\begin{array}{l}1962-3 \\
1964 \\
1965 \\
1966 \\
1967\end{array}$ & $\begin{array}{l}30 \\
45 \\
40 \\
57 \\
49\end{array}$ & $\begin{array}{l}0,26 \\
0,33 \\
0,38 \\
0,28 \\
0,21\end{array}$ & $\begin{array}{c}0,004 \\
<0,01 \\
0,01 \\
<0,01 \\
<0,01\end{array}$ & $\begin{array}{c}0,025 \\
0,02 \\
0,01 \\
0,02 \\
<0,01\end{array}$ & $\begin{array}{l}- \\
- \\
-\end{array}$ \\
\hline Pologne & $1969-70$ & 160 & 2,25 & $0,20(\gamma)$ & - & - \\
\hline U.R.S.S. & 1964 & - & 5,4 & - & - & - \\
\hline U.S.A. & $1966-67$ & - & 0,32 & 0,017 & 0,03 & 0,016 \\
\hline
\end{tabular}


Les départements expéditeurs sont les suivants : 01, 10, 14, 27, 28, $44,46,50,51,54,56,61,63,74,77,79,81,85,86,88,89$.

Les résidus observés sur les produits laitiers hors de France font apparaître l'importance relative des taux de résidus de DDT (et des dérivés) dont l'usage, malgré de nombreuses interdictions, est encore très répandu (voir tab. 3).

\section{B) RÉSIDUS DE DIPHÉNYLES POLYCHLORÉS}

Dans les quelques cas de contamination signalés aux Etats-Unis (dans le lait), les taux résiduels, rapportés à la matière grasse, ont varié de 0,1 à $0,3 \mathrm{ppm}$.

Un sondage important fait en Suède sur du lait de bovin et du beurre de grand commerce (Westoo et al., 1970) [6] a fait apparaître un haut degré de contamination par les PCB : en particulier tous les échantillons de beurre étaient pollués. Le taux résiduel moyen relevé était de 0,1 ppm pour les deux catégories d'échantillon.

\section{C) RÉsidus d'ANTHELminthiques}

Dans une enquête effectuée en Allemagne, des résidus de fasciolicides ont été mis en évidence dans le lait de bovins traités régulièrement contre la douve. Les résidus de ménichlopholane et d'oxyclosanide ont été décelés et dosés 10 à $15 \mathrm{j}$ environ après le traitement : maximum au $2-3^{\mathrm{e}} \mathrm{j}(0,08$ à $0,13 \mathrm{ppm}$ rapporté au lait total).

\section{II. - METABOLISME - ASPECT TOXICOLOGIQUE}

La présence de résidus de composés organo-chlorés dans les aliments a tout naturellement posé le problème de la toxicité à long terme pour l'homme et pour l'animal.

Avant d'évaluer cette toxicité, il est nécessaire de préciser un point important concernant l'élimination et le stockage des résidus. Il semble en effet que le " plateau d'équilibre " atteint, à long terme, entre l'absorption et l'élimination, ne soit pas constant, même avec une contamination ininterrompue. Le taux résiduel diminue progressivement dans l'organisme, que ce soit par suite d'une atténuation de l'absorption digestive ou d'une augmentation des processus enzymatiques de dégradation au niveau du foie.

D'autre part, il semble que lors de mobilisation brutale des graisses de l'organisme (stress physiologiques - amaigrissement - chocs infectieux ou chirurgicaux) il y aurait un stockage important des résidus dans le muscle et nul dans le système nerveux central (Findley et de Freitas, 1971) [7]. Il convient cependant de considérer la migration des résidus dans l'organisme comme un phénomène assez complexe, lié surtout aux interactions potentielles qui se produisent 
fréquemment entre certains composés : cas du DDT - PCB (Sodergren et Ulfstrand, 1972) [8].

Enfin, il faut indiquer qu'une corrélation étroite paraît exister entre les taux résiduels du lait et des tissus gras des carcasses chez les bovins (Stull et al., 1971) [9].

La même étude signale que les concentrations de résidus sont identiques dans toute la carcasse, quelque soit le lieu de prélèvement (résultat rapporté à la M.G.).

De toute façon, la sécrétion lactée demeure, pour les organochlorés les plus importants, la voie d'excrétion essentielle. C'est le cas pour l'époxyde de l'heptachlore (taux d'excrétion $\sim 40$ p. 100) et la dieldrine (taux d'excrétion 30 p. 100) (Hascoet, 1970) [10]. Le passage de l'HCHy (lindane), par contre, est extrêmement faible $(\sim 2$ p. 100) ce composé étant reconnu comme très instable « in vivo ».

Le cas des diphényles polychlorés est assez comparable. Des 10 p. 100 de la dose administrée qui sont éliminés dans les 7 premiers jours par les urines, les fécès et le lait, 7,35 p. 100 le sont par cette dernière voie. Là encore, le lait est la principale voie d'excrétion.

Les anthelminthiques enfin s'accumulent très peu en général dans les tissus. Les fasciolicides, qui nous intéressent semblent cependant s'éliminer plus lentement, leur persistance étant néanmoins peu importante (15 à $20 \mathrm{j})$.

Compte tenu de ces bilans métaboliques, assez différents selon les composés en cause, l'évaluation du risque pour l'homme a été calculée, d'une part grâce aux observations des cas d'intoxications, la plupart du temps aigus et assez rares, d'autre part grâce à un très grand nombre d'expérimentations sur l'animal.

Si le mécanisme d'action biochimique est encore assez mal connu, de nombreuses données existent par contre sur les effets à long terme des composés qui nous intéressent.

\section{1) Actions sur les fonctions hépatiques}

L'administration de pesticides organo-chlorés aux mammifères augmente le poids du foie (par rapport au poids total), la synthèse des protéines des microsomes et surtout des enzymes, oxydants ou réducteurs.

Cette dernière " activation » physiologique est responsable de la dégradation " in situ " d'un grand nombre d'agents pharmacologiques : antipyrine, phénobarbital, etc.

Les cyclodiènes, en particulier, peuvent être considérés avec leurs époxydes correspondants, comme des stimulants de l'oxydation microsomale. La concentration sans effet est comprise entre 1 et 5 ppm sur le rat. 
L'heptachlore et l'aldrine apparaissent comme des cosubstrats sur le même enzyme et sont compétitivement inhibés par la dieldrine et l'époxyde de l'heptachlore, respectivement.

Les études actuelles ne permettent malheureusement pas encore de connaître avec précision le métabolisme ultérieur des époxydes de ces composés dans les microsomes du foie.

La pathogénèse des intoxications par l'HCB est encore mal connue. Ce composé paraît avoir une action directe sur les cellules hépatiques : la stimulation de l'activité enzymatique (toujours au niveau des microsomes) se traduirait par une production accélérée de l'acide delta-amino-levulinique synthétase. Aucune action sur la coquille de l'ouf. Pas de lésions apparentes du système nerveux.

Les altérations causées par les contaminations dues aux PCB se rapprochent assez sensiblement de celles qui sont observées avec l'HCB. La porphyrie chimique est la conséquence d'une induction de l'acide delta-amina-levulinique synthétase (dans le microsome) par le polluant.

\section{2) Action endocrinienne}

1) Action cestrogène: Dès 1953, il était reconnu que l'administration de faibles doses d'aldrine $(10 \mathrm{ppm})$ perturbait l'œstrus chez le rat.

Levin et al. (1968) [11], puis Welch et al. (1969) [12] démontrent nettement l'action œstrogène de l'op'DDT chez le rat. Depuis les recherches ont été approfondies et il est possible de considérer maintenant comme admises les conclusions suivantes :

a) Pesticides organo-chlorés : L'action œstrogène, révélée sur utérus de rats impubères est la suivante, par ordre décroissant : op'DDT > méthoxychlore (op' + pp') > pp'DDT et pp' méthoxychlore

Il faut noter ici que l'activité de ces composés est de l'ordre du $\mathrm{mg}$, alors que la plupart des œstrogènes ont une gamme de réponse voisine du $1 / 10 \mathrm{du} \mu \mathrm{g}$.

b) Diphényles polychlorés : Seuls les composés les moins chlorés (jusqu'à 48 p. 100) présentent une action œstrogène, plus faible d'ailleurs que celle du DDT.

Il faut préciser ici que l'isomère op' du DDT est rapidement transformé " in vivo " en pp' DDT. Ce processus de détoxification naturel est très important. Le corollaire ne paraît malheureusement pas s'appliquer aux PCB dont la grande stabilité suppose une activité œstrogène continue.

2) Autres actions : Par ailleurs, des expérimentations réalisées sur plusieurs espèces de mammifères, il ressort que de faibles doses de DDT et de dieldrine peuvent perturber le métabolisme de la testostérone et de la progestérone par stimulation de l'activité enzymatique 
du foie. C'est ce mécanisme qui a été proposé pour expliquer la diminution de l'épaisseur des coquilles des œufs de rapaces et de certains oiseaux marins (altération du métabolisme du calcium) d'où leur disparition.

Il semble également que le pp'DDE, métabolite majeur du DDT chez l'oiseau, joue un rôle analogue.

Enfin, l'op'DDD (et à un degré moindre le pp'DDD) métabolites majeurs du DDT chez les mammifères, présentent une action inhibante sur le cortex surrénal (action antistéroïdienne - diminution de la sécrétion corticostéroïdienne). Cette faculté a été d'ailleurs mise à profit par l'utilisation thérapeutique de l'op'DDD dans le traitement du syndrome de Cushing et de certaines formes de carcinome surrénal.

Les diphényles polychlorés ont été aussi identifiés comme ayant un pouvoir activateur vis-à-vis des enzymes hépatiques, en particulier chez les oiseaux (métabolisation de l'œstradiol).

Récemment, les PCB ont été reconnus comme exerçant un effet anti-stéroïdien (inhibition des effets de type androgène) chez l'oiseau (concentration d'essai $200 \mathrm{ppm}$ pendant 13 semaines). Des études sont en cours pour déterminer les conséquences, apparemment assez importantes, des contaminations par les PCB sur la reproduction des oiseaux et des animaux domestiques en général.

Ajoutons que la thyroïde paraît également sensible aux contaminations par certains composés organo-chlorés (pp'DDT notamment) : manifestations d'hyperthyroïdisme à faible dose, d'hypothyroïdisme à forte dose (chez l'oiseau).

3) EMBRYotoxicité - ACTION TÉRATOGÈne : Il a été démontré que le fœtus et le nouveau-né sont extrêmement sensibles aux contaminations par la plupart des produits chimiques organiques de synthèse. La mortalité peut se produire à des niveaux de concentration bien au-dessous de ceux pouvant présenter un danger réel pour le sujet adulte.

La barrière placentaire est facilement traversée par la grande majorité des composés organo-chlorés, que ce soit chez le lapin, la souris, le chien, le rat et l'homme. Des teneurs de DDT aussi élevées que 13,5 ppm et $26 \mathrm{ppm}$ ont été retrouvées respectivement dans le placenta humain et le cordon ombilical. Le fotus, in utéro, concentre les résidus de certains composés organo-chlorés dès la $4^{\text {e }}$ semaine de gestation. Le lait maternel peut apporter également sa contribution à la contamination du nouveau-né : les résidus de la plupart des pesticides organo-chlorés, y compris l'HCB, et récemment les $\mathrm{PCB}$, ont été décelés dans le lait maternel humain. Les taux résiduels moyens de DDT dans le lait maternel humain, aux Etats-Unis (soit 0,05 à 0,26 ppm en 1969), sont nettement plus élevés, à la même période que ceux retrouvés dans le lait de vache ou de brebis. 
Le risque particulier du foetus ou du nouveau-né est dû à quatre raisons principales :

1) La circulation des résidus est plus importante dans un organisme où les dépôts lipidiques ne sont pas encore entièrement formés.

2) Absence de système enzymatique hépatique fonctionnel, pas d'élimination des corps chimiques étrangers.

3) Fonction rénale réduite, pas de transport de molécules organiques.

4) Barrière hémo-méningée (du système nerveux central) peu développée.

L'action tératogène, de son côté, s'exerce en général sur le foetus durant l'organogénèse (fin $1^{\mathrm{e}}$ semaine jusqu'à la $12^{\mathrm{c}}$ semaine chez l'homme). Les malformations congénitales semblent exceptionnelles avec les composés organo-chlorés, bien qu'une certaine embryotoxicité ait été reconnue à la dieldrine, au chlordane et l'op'DDD. Il n'y a pas, de toute façon, d'augmentation significative des anomalies.

4) EfFets CancÉRogènes, interactions : Les effets cancérogènes ou précancérogènes pouvant résulter de la présence de résidus de composés organo-chlorés, chez les mammifères (et chez l'homme) n'ont pas été démontrés encore aujourd'hui avec netteté.

Dans une importante étude chez la souris, Innes (1969) trouve que le pp'DDT augmente la fréquence des hépatomes au taux contaminant de $46,4 \mathrm{mg} / \mathrm{kg}$ [13]. Ont été récemment considérés comme des cancérogènes potentiels les cyclodiènes avec leurs époxydes. Il convient cependant de préciser que toutes ces expérimentations ont été réalisées avec des doses relativement élevées et que le test " hépatome " chez la souris a perdu aujourd'hui une grande part de sa signification pathologique. De toute façon, l'effet cancérogène chez l'homme reste à démontrer, l'extrapolation de résultats expérimentaux sur animaux demeurant irréalisable dans presque tous les cas (rapport Mrak, 1969) [14].

Actuellement, aucune donnée précise n'est connue sur un effet cancérogène possible des PCB chez l'homme ou chez l'animal.

Terminons l'étude des principales actions (ou effets des composés organo-chlorés sur l'organisme animal (ou humain) en ajoutant que de nombreuses expérimentations se poursuivent sur les interactions de nombreuses matières actives de structures parfois très voisines : $\mathrm{DDT} / \mathrm{PCB}$ - HCB/PCB - dieldrine/DDT, etc. Ces problèmes sont très importants, ils conditionnent l'évaluation des risques encourus par un composé et peuvent modifier le calcul d'une D.J.A.

En résumé, il est possible actuellement de considérer comme acquis les points suivants :

1) Les informations les plus intéressantes concernent les effets à long terme consécutifs aux inductions enzymatiques des microsomes 
TABLEAU 4

\begin{tabular}{|c|c|c|c|c|c|c|c|}
\hline \multirow{2}{*}{$\begin{array}{l}\text { FAO/OMS } 1970 \\
\text { D.J.A. } \\
\mu \mathrm{g} / \mathrm{kg} / \text { jour }\end{array}$} & & \multicolumn{6}{|c|}{$\begin{array}{c}\text { Consommation moyenne } \\
\mu \mathrm{g} / \mathrm{kg} / \text { jour }\end{array}$} \\
\hline & & $\begin{array}{l}\text { France } \\
1971\end{array}$ & Cons $/ \mathrm{DJA}$ & $\underset{1968}{\text { Hollande }}$ & Cons /DJA & $\begin{array}{l}\text { U.S.A. } \\
1968\end{array}$ & Cons /DJA \\
\hline Dieldrine-Aldrine & 0,1 & 0,060 & 0,60 & 0,025 & 0,25 & 0,016 & 0,16 \\
\hline Heptachlore-Epoxyde-Heptachlore & 0,5 & 0,067 & 0,13 & 0,01 & 0,02 & 0,016 & 0,03 \\
\hline $\mathrm{HCH}$ & - & 0,194 & - & - & - & - & - \\
\hline $\mathrm{HCH}_{\gamma}$-Lindane & 12,5 & 0,027 & 0,002 & 0,008 & 0,0006 & $\mathrm{~T}$ & - \\
\hline DDT-DDE-DDD & $\begin{array}{l}5 \\
\text { (sous réserve) }\end{array}$ & $\mathrm{T}$ & & 0,065 & 0,13 & 0,180 & 0,30 \\
\hline НСВ & $\begin{array}{l}0,6 \text { sous réserve } \\
\text { (localisé) }\end{array}$ & & & & & & \\
\hline \multicolumn{8}{|l|}{ Consommation: } \\
\hline \multicolumn{4}{|c|}{$\begin{array}{l}\text { Pour la France : résultats exprimés en fonction des prévisions } 1970 \text {. } \\
\text { Enquête I.N.S.E.E. } 1967 \rightarrow 0,62 \mathrm{~g} / \mathrm{kg} / \mathrm{jour} \text {. } \\
\text { Prévisions } 1970 \rightarrow 0,67 \mathrm{~g} / \mathrm{kg} / \mathrm{jour} \text {. }\end{array}$} & \multicolumn{4}{|c|}{$\begin{array}{l}\text { Comparaison consommation Hollandaise ou } \\
\text { Américaine : } \\
0,54 \mathrm{~g} / \mathrm{kg} / \text { jour. }\end{array}$} \\
\hline
\end{tabular}

Consommation :

Pour la France : résultats exprimés en fonction des prévisions 1970 Prévisions $1970 \rightarrow 0,67 \mathrm{~g} / \mathrm{kg} / \mathrm{j}$ jour 
du foie par la plupart des composés organo-chlorés (en particulier les cyclodiènes). Par ailleurs, la formation de photoisomères in vivo, plus toxiques que les molécules mères, est l'objet actuellement de nombreux travaux.

2) Le risque, pour l'animal ou pour l'homme, est prépondérant pour tout ce qui touche les phénomènes de la reproduction.

Il est évident ainsi que la présence de résidus de pesticides organochlorés (Goursaud et al., 1971) [15], de diphényles polychlorés (Acker et al., 1970) [16] dans le lait de femme, peut causer certaines inquiétudes aux hygiénistes spécialisés.

Toutes les données obtenues justifient la procédure d'évaluation du risque et sa prévention : utilisation de la D.J.A. (dose journalière acceptable), d'un coefficient de sécurité (100 en général), de la C.M.A. (concentration maximale autorisable du résidu) dans l'aliment, de la consommation journalière moyenne.

En ce qui concerne le lait et les produits laitiers, il est intéressant d'évaluer le rapport consommation moyenne journalière sur D.J.A. (voir tab. 4). Il est intéressant de constater ainsi qu'en France comme dans de nombreux pays (Hollande, U.S.A., Grande-Bretagne), parmi les résidus de pesticides organo-chlorés, la dieldrine présente le rapport le plus élevé.

\section{III. - ASPECT ECONOMIQUE}

L'abaissement des niveaux de contamination en France, a contribué certainement à l'augmentation des exportations de produits laitiers (notamment des fromages : + 46 p. 100 en 1971 par rapport à 1970).

Les normes des pays importateurs (voir tab. 5) sont encore néanmoins un obstacle aux échanges internationaux.

Les difficultés actuelles, en France, proviennent essentiellement des taux résiduels de l'époxyde de l'heptachlore et, à un degré moindre, de ceux de la dieldrine. Ces taux devraient nécessairement baisser dans les mois à venir par suite de nouvelles mesures réglementaires qui doivent entrer en vigueur prochainement.

Notons que de nombreux composés organo-phosphorés sont déjà utilisés, comme produits de remplacement, à la fois dans les locaux de stabulation et dans certains traitements phyto-sanitaires particuliers.

\section{IV. - MESURES DE PREVENTION}

Des mesures administratives nouvelles vont s'ajouter prochainement à des textes réglementaires importants déjà en vigueur. 
TABLEAU 5

Tolérances administratives en résidus de pesticides dans le lait et les produits laitiers (exprimées en mg/kg de M.G.)

\begin{tabular}{|c|c|c|c|c|c|}
\hline Organochlorés & FAO/OMS & U.S.A. & Canada & Yougoslavie & Suisse \\
\hline HCB seul & 0,30 & 0,30 & & & \\
\hline $\begin{array}{c}\mathrm{HCH} \alpha \\
\beta \\
\gamma \\
\text { Total } \alpha+\beta+\gamma\end{array}$ & $\gamma 0,10$ & 0,30 & $\begin{array}{r}* \beta 0,10 \\
\gamma 0,30 \\
0,30\end{array}$ & $\gamma 0,20$ & 0,10 \\
\hline $\begin{array}{l}\text { Heptachlore }+ \text { Heptachlore } \\
\text { Epoxyde }\end{array}$ & 0,15 & 0,30 & 0,10 & & 0,125 \\
\hline Aldrine + Dieldrine & 0,15 & 0,30 & 0,10 & 0,125 & 0,125 \\
\hline $\mathrm{DDT}+\mathrm{DDD}+\mathrm{DDE}$ & 1,25 & 1,00 & 1,00 & 0,125 & 0,125 \\
\hline Chlordane & & 0,10 & 0,10 & & \\
\hline
\end{tabular}

* La tolérance administrative pour l'isomèreß a été relevée à $0,30 \mathrm{mg} / \mathrm{kg}$ (M.G.) depuis le $1^{\text {er }}$ juin 1972. 


\section{1) Réglementation en vigueur (en France)}

- Arrêté du 15 octobre 1969 : Interdiction d'emploi de 1'HCH (y compris lindane) de l'aldrine, de la dieldrine, et du DDT, pour la désinsectisation des étables et de tous locaux servant au logement des animaux domestiques ou à la préparation de leur nourriture.

- Décret du 30 juillet 1971 : Interdiction de vente des denrées ou boissons destinées à l'alimentation humaine ou animale, contenant une teneur en résidus de produits utilisés en agriculture et en élevage (dont les pesticides) présentant un danger pour la santé humaine, même si le danger n'apparaît qu'après une longue période de consommation.

- Arrêté du 6 août 1971 : Interdiction de vente, pour la consommation humaine, de viande ou de sous-produits des animaux ayant reçu des pesticides organo-chlorés, à la suite de traitements thérapeutiques vétérinaires, pendant une période de 3 ans au moins, en raison de la persistance des résidus.

- Circulaires d'application des 22 et 28 juillet 1970 , du 14 octobre 1971 : Relatives aux conditions de traitement des locaux de stabulation ou de stockage des aliments.

\section{2) Réglementation à venir (en France)}

- Circulaire d'application de l'arrêté du 6 août 1971 : Désignation des pesticides organo-chlorés : $\mathrm{HCH}$ (tous les isomères), aldrine, dieldrine, chlordane, heptachlore, DDT, DDD, toxaphène, endosulfan.

- Interdiction totale de vente (fin juin 1972) et d'emploi ( $1^{\text {er }}$ octobre 1972) des cyclodiènes chlorés en agriculture (vise l'heptachlore, le chlordane, l'aldrine et la dieldrine).

- Dans le cadre européen (C.E.E.), des commissions spécialisées établissent actuellement des projets de directive visant à l'établissement de Normes de tolérances pour les résidus de pesticides (y compris organo-chlorés) dans les denrées d'origine animale ou végétale (alimentation de l'homme ou alimentation de l'animal).

Ces normes, garanties essentielles de la santé du consommateur, doivent rétablir des conditions normales d'échanges (importations, exportations) vis-à-vis des pays déjà pourvus de ce système de protection).

Un exemple intéressant nous est fourni par les Normes de tolérance de la Food and Drug Administration (U.S.A.) (voir tab. 6) qui sont imposées à une grande variété d'aliments pour l'homme. Ces normes sont basées sur le principe dit des « listes positives » principe qui est maintenu pour les Normes Européennes en projet.

Ajoutons qu'en ce qui concerne les diphényles polychlorés, il n'existe pas de Normes de tolérances en France. Aux Etats-Unis, une tolérance provisoire a été établie récemment pour le lait et les produits laitiers soit 2,5 ppm (rapporté à la M.G.). 
Code of Federal Regulations F.D.A. (U.S.A.), janvier 1970 - Tolérances en ppm (mg/kg) - Insecticides organo-chlorés

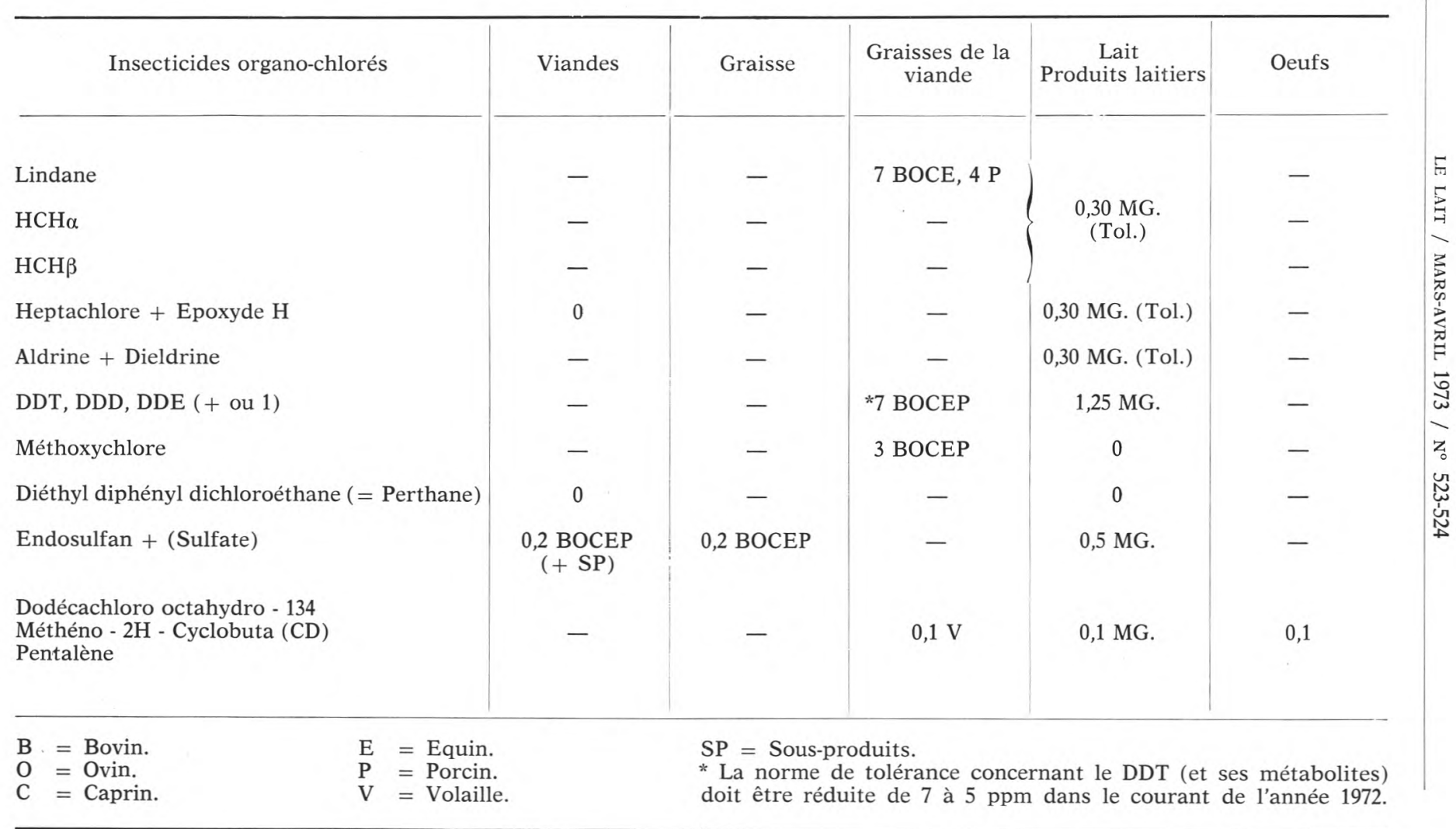


A notre connaissance, aucune réglementation ne vise actuellement les résidus d'anthelminthiques dans le lait et les produits laitiers.

\section{CONCLUSION}

Les résidus de pesticides organo-chlorés constituent actuellement les principaux contaminants du lait et des produits laitiers. Par ailleurs, les résidus de certains polluants d'origine industrielle dont les diphényles polychlorés et de quelques anthelminthiques utilisés en thérapeutique vétérinaire ont été récemment mis en évidence dans le lait en Allemagne et aux Etats-Unis.

En France, ces deux dernières années, après les premières mesures prises contre la pollution du lait et des produits laitiers par les résidus de pesticides organo-chlorés, une nette amélioration s'est manifestée notamment sur les résidus d'H.C.H., principe polluant.

Des problèmes demeurent, liés notamment aux taux résiduels de l'époxyde de l'heptachlore et de la dieldrine. Les processus de contamination sont connus; ils s'exercent par la voie des aliments du bétail :

1) Par les fourrages frais, les céréales (en général peu pollués mais qui peuvent être accidentellement contaminés dans les locaux de stockage).

2) Par les betteraves, le maïs : responsables de la contamination par l'époxyde de l'heptachlore.

3) Par les aliments concentrés et les tourteaux (surtout d'arachide) pollués soit à l'origine, soit au cours de la préparation.

Pour l'instant, la pollution des laits ou des produits laitiers par d'autres composés organo-chlorés (PCB, anthelminthiques) ne semble pas préoccupante, tout au moins en France sur le plan général. L'attention des laboratoires de contrôle doit néanmoins être attirée sur l'éventualité de la présence de tels résidus (surtout les PCB) dans les échantillons de lait soumis à leur inspection.

Les nouvelles mesures administratives, l'établissement de Normes de tolérances à l'échelon Européen, les dispositions prévues dans le projet de loi sur la Pharmacie Vétérinaire et la politique de surveillance des aliments pour animaux, permettent d'envisager enfin une solution aux derniers problèmes créés par la pollution des produits laitiers par les résidus de pesticides organo-chlorés.

\section{S u m m a r y}

Chlorinated pesticides are the major contaminants, in milk, butter, and cheese. In the mean time, other chlorinated polluants 
have been detected, i.e. polychlorobiphenyls, and also some newer anthelmintics used in veterinary therapeutic.

Last two years, in France, a clean improvement appeared for B.H.C. residues. On the other hand heptachlor-epoxyde and dieldrin residues always have the same level of contamination. For this two compounds, the residues found in milk and milk products can be traced back to commercial feed mixtures and concentrates (beets waste) and also in cow-forage.

Some pesticides such as aldrin, heptachlor, methoxychlor, and in most cases DDT or metabolites, were not found. Numerous samples contained hexachlorobenzene $(\mathrm{HCB})$ residues but with very low contamination level. PCB or anthelmintic residues were not found in the control samples.

The acceptable daily intake (A.D.I.) of these pesticides is compared with the real intake on the basis of milk fat consumption. Awaiting European Tolerance (C.E.E.) levels for pesticide residues in feed products, new regulations and official recommendations are showed and discussed.

\section{Bibliographie}

[1] Mestres (R.) (1966). - Bull. INSERM, t. 21, n 3, p. 445.

[2] Thieulin (G.), Pantaleon (J.), Richou (L.), Cumont (G.) (1966). - Bull. Acad. Nat. Méd., 150, p. 478.

[3] VIEL et coll. (1967). - Congrès Intern. Protection des Plantes, Vienne, B. IV, 27.

[4] Jensen (S.) (1966), - New Scient, 32, p. 612.

[5] Heeschen (W.), Tolle (A.), Bluthgen (A.) (1971). - A.I.D. 53, Bonn. Bad Godesberg 1, Heerstrasse 124, Heft 173, p. 18.

[6] Westoo (G.), Noren (K.), Andersson (M.) (1970). - Var foda, 2-3, p. 10.

[7] Findley (G.M.), DE Freitas (A.S.W.) (1971). - Natuie, 229, p. 63.

[8] Sodergren (A.), Ulfstrand (S.) (1972). - Ambio, 1, p. 36.

[9] Stull (J. W.), Brown (W. H.), Whiting (F. M.) (1971). - Bull. of Environ. contam. toxicol., 6, p. 568.

[10] Hascoet (M.) (1970). - B.T.I., 252, p. 521.

[11] Levin (W.), Welch (R. M.), Conney (A. H.) (1968). - Fed Proc, 27, p. 649.

[12] Welch (R. M.), Levin (W.), Conney (A. H.) (1969). - Toxicol. and appl. pharmacol., 14 , p. 358 .

[13] InNes (J. R. M.) .et coll. (1969). - J. of the Nat. Can. Inst., 42, p. 1101.

[14] MRAK, Dr Emil. M. - Report of the secretary's Commission on Pesticides and their relationship to environmental Health (U.S.A.), dept. of health, education and welfare. Washington.

[15] Goursaud (J.), Luquet (F. M.), Casalis (J.). (1971). - Le Lait, 508, p. 559.

[16] ACKER (L.), SchULTE (E.) (1970). - Naturwissenschaften, 57, p. 497. 